\title{
Experimental study of the correlation between the elastic modulus of polymer composite materials and the velocity of ultrasonic waves
}

\author{
Dmitrii Chulkov ${ }^{1, *}$, Alexander Terekhin ${ }^{1}$, Alexander Dumansky ${ }^{2,3}$, and Maxim Tipikin ${ }^{1}$ \\ ${ }^{1}$ JSC «ORPE «Technologiya» named after A. G. Romashin», 249031 Obninsk, Russia \\ ${ }^{2}$ Mechanical Engineering Research Institute of Russian academy of sciences, Materials Sciences \\ Department, 101990 Moscow, Maly Kharitonievsky per., 4, Russia \\ ${ }^{3}$ Bauman Moscow State Technical University, 105005, Moscow, 2-ya Baumanskya str., Russia
}

\begin{abstract}
This article presents the results of an experimental study of the relationship of acoustic parameters (propagation velocity) of ultrasonic waves with elastic characteristics (elastic modulus) of polymer composite materials. Significant regression dependences of the correlation between the velocity of ultrasonic won and the modulus of elasticity of a composite material based on fiberglass with an epoxy and phenol-formaldehyde binder have been constructed.
\end{abstract}

\section{Introduction}

To ensure the high quality of critical structural elements made of polymer composite materials, it is necessary to use modern methods and means of non-destructive testing. An important area of non-destructive quality control of materials and structures made of polymer composite materials is the determination of physical and mechanical characteristics, and in particular, the elastic modulus. As is known, the dynamic modulus of elasticity of a material is related to the velocity of ultrasonic waves by a functional dependence $[1,2]$ :

$$
E=\frac{\rho C_{l}^{2}(1+\mu)(1-2 \mu)}{(1-\mu)}
$$

where $\rho$ is density of the material; $C_{l}$ is velocity of longitudinal ultrasonic waves; $\mu$ is Poisson ratio.

However, due to the pronounced anisotropy of the properties of composite materials, including acoustic ones $[3,4]$, the determination of the elastic modulus by the non-destructive ultrasonic method is possible only by the previously constructed regression dependences $[2,3,5]$. Based on the above, the aim of this work is to conduct an experimental study of the relationship between acoustic parameters (propagation velocity) of ultrasonic waves with elastic characteristics (elastic modulus) of polymer composite materials using the example of fiberglass plastics with unidirectional and angle-ply reinforcement.

* Corresponding author: DIChulkov@gmail.com 


\section{Samples and research methods}

For experimental studies, 2 panels were made, reinforced with TC 8/3-K-TO fiberglass. Panel characteristics are shown in Table 1.

Table 1. Panel characteristics.

\begin{tabular}{|c|l|l|l|}
\hline Panel No & Binder & Layout of fiberglass & Size, $\mathrm{mm}$ \\
\hline 1 & Phenol-formaldehyde & {$[0 / \pm 33 / \pm 63 / 0]$} & $605 \times 335 \times 1,5$ \\
\hline 2 & Epoxy (EDT-10) & unidirectional & $320 \times 260 \times 1,9$ \\
\hline
\end{tabular}

Samples were made from panels 1 and 2 to determine the flexural and tensile modulus. Determination of the elastic modulus by the destructive method was carried out in accordance with the regulatory documents $[6,7]$.

Before determining the modulus of elasticity by the destructive method, the velocities of ultrasonic waves in the plane of the sample were measured in each sample. To carry out measurements, piezoelectric transducers with a resonant frequency of $1 \mathrm{MHz}$ with conical prisms were developed and manufactured, which make it possible to measure the speed of ultrasonic waves along the layers of fiberglass at different sounding bases (distance between the exciting and receiving transducers). A digital ultrasonic flaw detector was used as a generator and receiver of ultrasonic waves. The measurement circuit and the appearance of the transducers are shown in Figure 1.

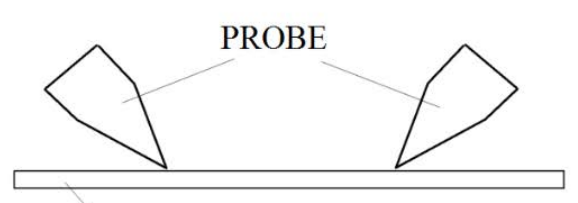

TEST PIECE

a

Fig. 1. Measurement illustration (a), external view of piezoelectric transducers with conical prisms and fixation device (b).

To make an estimate of the correlation between the studied parameters, the Pearson correlation coefficient was used, calculated by the formula [8]:

$$
r=\frac{s_{x y}}{\sqrt{s_{x} S_{y}}}
$$

where $S_{x}=\sum\left(x_{i}-\bar{x}\right)^{2}$ и $S_{y}=\sum\left(y_{i}-\bar{y}\right)^{2}$ are sums of squares of regression residuals parallel to the axes $X$ and $Y ; S_{x y}=\sum\left[\left(x_{i}-\bar{x}\right)\left(y_{i}-\bar{y}\right)\right]$ is sum of squares of regression residuals perpendicular to the straight line, $S_{x y}, \bar{x}$ and $\bar{y}$ are arithmetic means over all $x_{i}$ and $y_{i}$. To assess the quality of regression models, the $R^{2}$-squared values was used, calculated by the formula:

$$
R^{2}=1-\frac{\sum\left(y_{i}-\hat{y}_{i}\right)^{2}}{\sum\left(y_{i}-\bar{y}\right)^{2}}
$$

where $\hat{y}_{i}$ is the value obtained from the regression equation, $\bar{y}$ - mean value.

To assess the quality of the multi-parameter regression model, we used the corrected coefficient of determination, determined by the ratio: 


$$
R_{a d j}^{2}=1-\left(1-R^{2}\right) \frac{n-1}{n-k-1},
$$

where $R_{a d j}^{2}$-squared values; $n$ is sample size; $k$ is number of model parameters.

\section{Data analysis}

Figure 2 shows the experimental dependence of the modulus of elasticity in bending on the velocity of ultrasonic waves in the plane of the samples made from panel 1 . The samples cut from the panel along the direction of the weft threads are shown in green (square marker), and red (round marker) - along the direction of the warp threads.

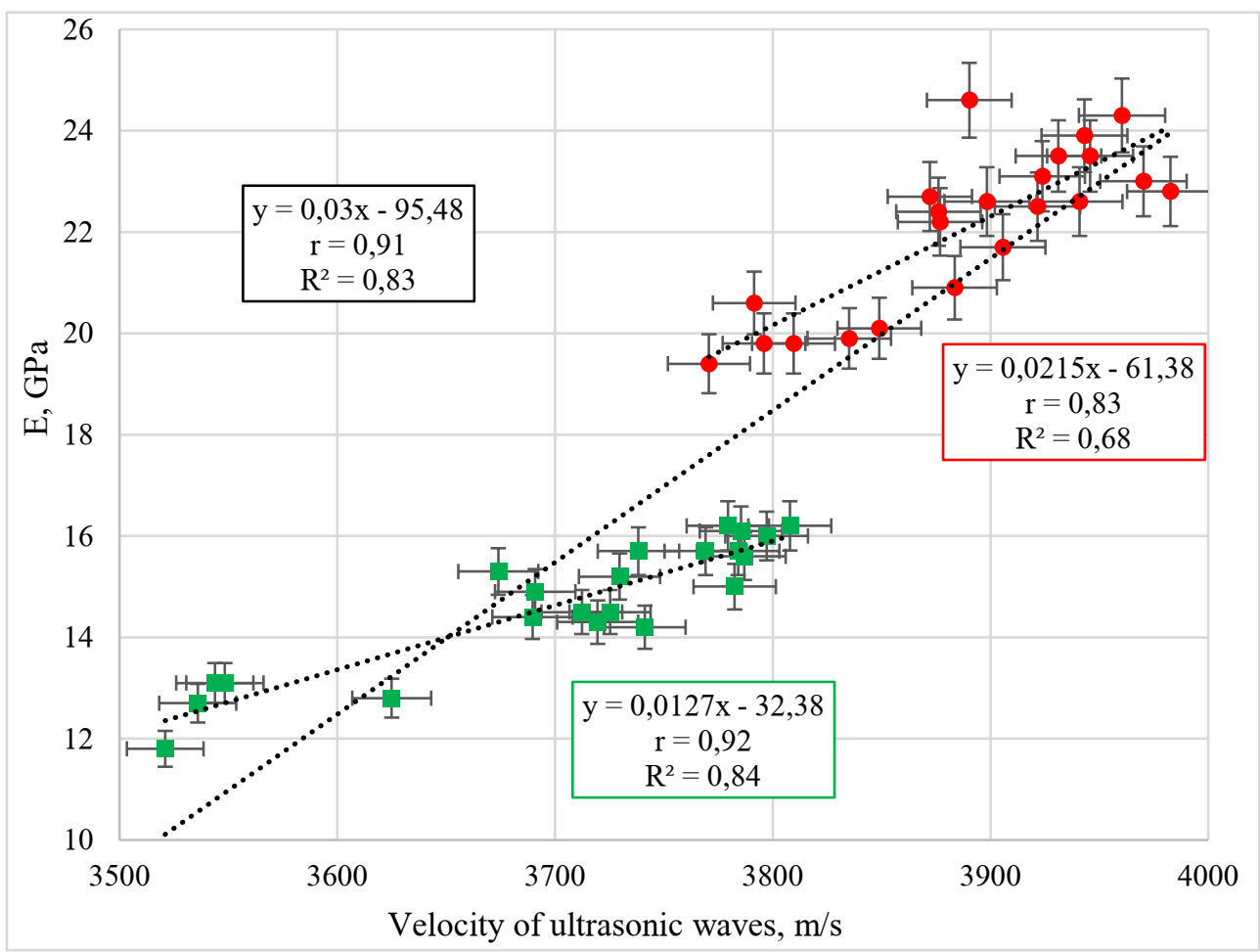

Fig. 2. Dependence of the elastic modulus in bending on the speed of ultrasonic waves in samples from panel 1 (calculation $[0 / \pm 33 / \pm 63 / 0]$.

In Figure 2 is shown that the modulus of elasticity of the samples is $11.8-16.2 \mathrm{GPa}$ in the weft direction and 19.4 - 24.6 GPa in the warp direction. In this case, the speed of ultrasonic waves varies in the range of $3520-3810 \mathrm{~m} / \mathrm{s}$ for samples cut in the direction of the weft, and $3770-3980 \mathrm{~m} / \mathrm{s}$ for samples cut in the direction of the warp. Analysis of the obtained experimental data shows that for the material of samples from panel 1, there are correlation between the modulus of elasticity in bending and the speed of ultrasonic waves ( $\mathrm{r}$ $=0.92$ for the weft, $r=0.83$ for the warp, $r=0.91$ for all samples), which indicates the possibility of assessing the elastic modulus of the material by the speed of ultrasonic waves.

In Figure 3 is shown a graph of the dependence of the modulus of elasticity in bending on the speed of ultrasonic waves in the plane of the samples made from panel 2 (epoxy binder, unidirectional layout). Patterns cut along the direction of the weft threads are marked in green (square marker), and in red (round marker), along the direction of the warp threads. 


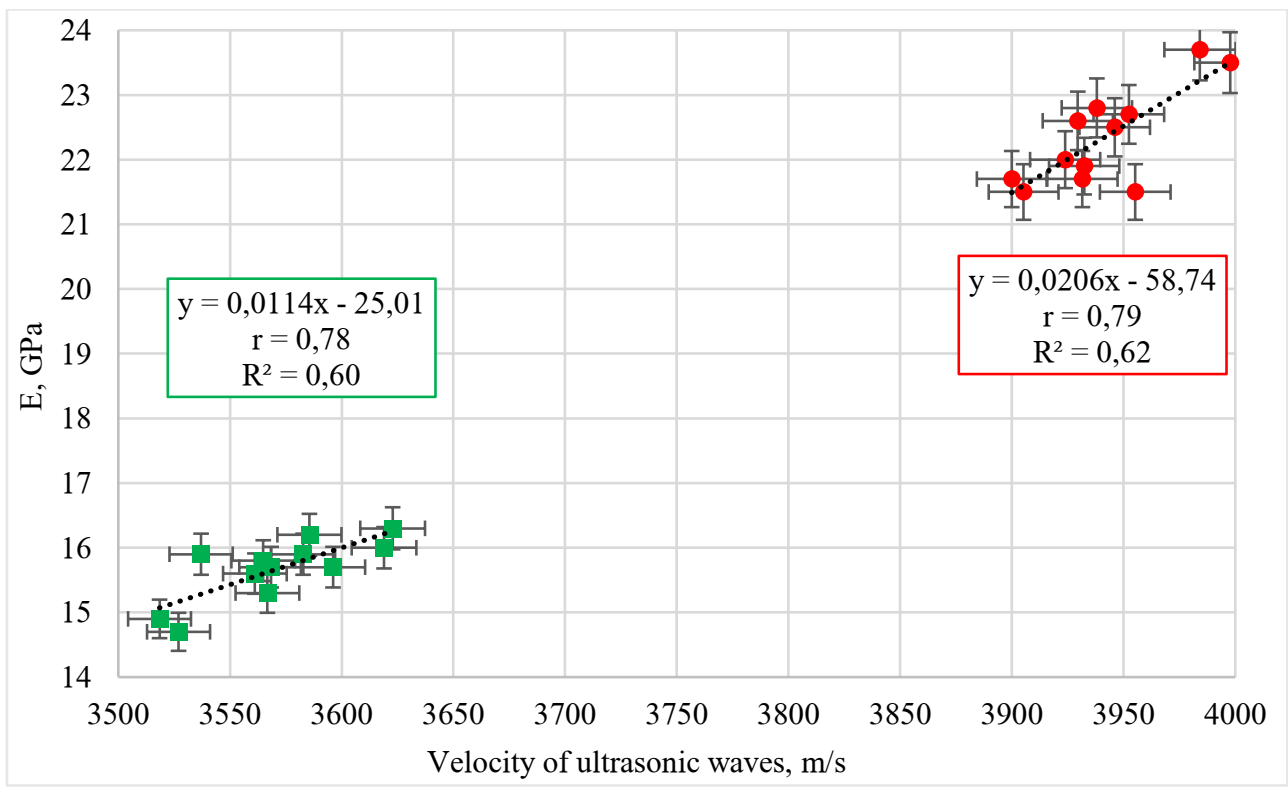

Fig. 3. Dependence of the modulus of elasticity in bending on the velocity of ultrasonic waves for samples from panel 3 (epoxy binder, unidirectional).

In Figure 3 is shown that the modulus of elasticity of the samples is in the range of $14.5-16.5 \mathrm{GPa}$ in the direction of the weft and $21-24 \mathrm{GPa}$ in the direction of the warp. In this case, the speed of ultrasonic waves varies in the range of $3500-3640 \mathrm{~m} / \mathrm{s}$ in the direction of the weft and $3880-4020 \mathrm{~m} / \mathrm{s}$ in the direction of the warp. Analysis of the obtained experimental data shows that for the material of panel 2 (epoxy binder, unidirectional layout), there is a relationship between the flexural modulus and the speed of ultrasonic waves $(\mathrm{r}=0.77$ for a weft, $\mathrm{r}=0.79$ for a warp). A relatively weak regression relationship can be caused by a small range of changes in the elastic modulus of the samples under study.

In Figure 4 is presented the dependence in plane of the tensile modulus of elasticity on the velocity of ultrasonic waves. Patterns cut along the direction of the weft threads are marked in green (square marker), and in red (round marker), along the direction of the warp threads. 


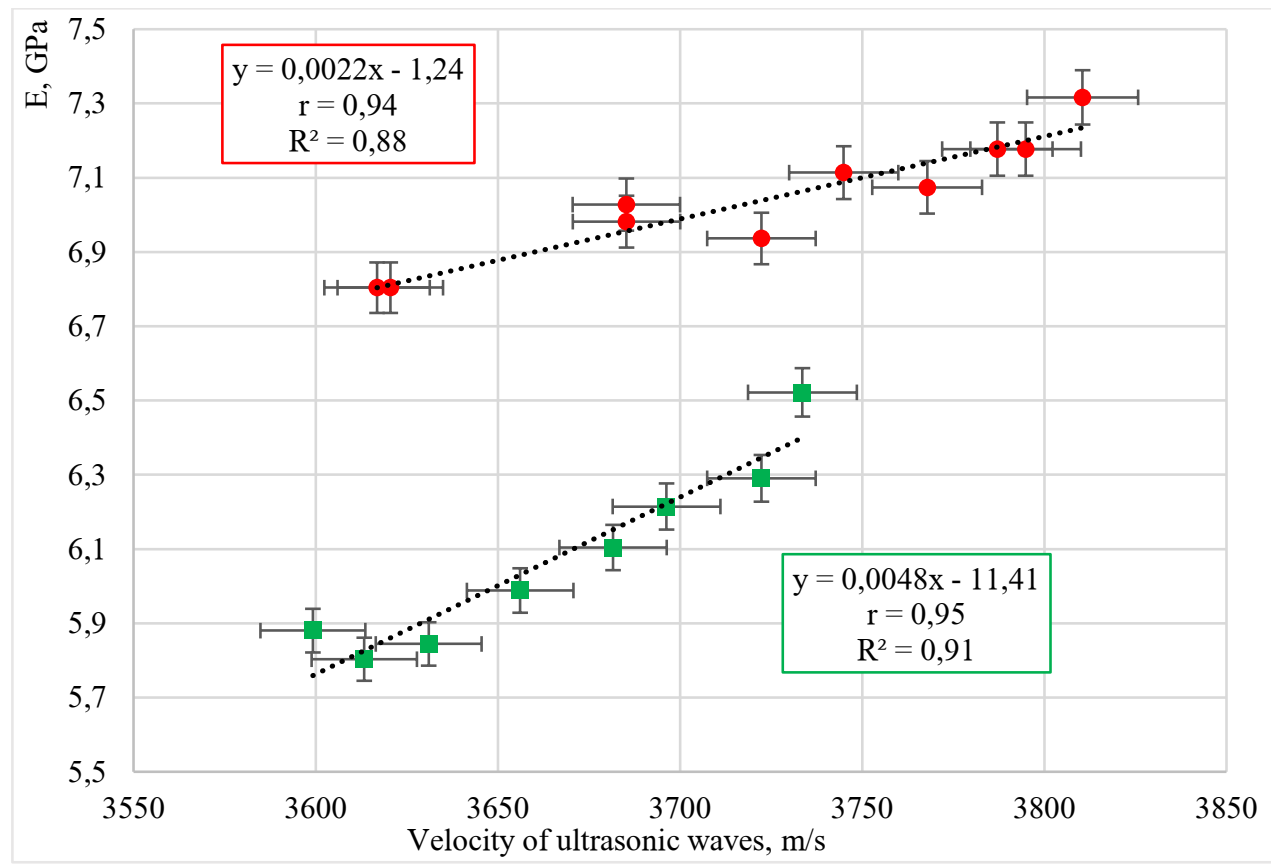

Fig. 4. The dependence of the longitudinal tensile modulus of elasticity on the velocity of ultrasonic waves of glass for samples from panel 1 (phenol-formaldehyde binder, lay-up $[0 / \pm 33 / \pm 63 / 0]$ ).

In Figure 4 is shown that the modulus of elasticity of the samples is in the range of $5.8-6.5 \mathrm{GPa}$ in the direction of the weft and $6.8-7.3 \mathrm{GPa}$ in the direction of the warp. In this case, the speed of ultrasonic waves varies in the range of $3600-3730 \mathrm{~m} / \mathrm{s}$ in the direction of the weft and $3620-3810 \mathrm{~m} / \mathrm{s}$ in the direction of the warp. Analysis of the obtained experimental data shows that for the material of panel 1 , there is a relationship between the tensile modulus of elasticity and the speed of ultrasonic waves $(r=0.95$ for the weft, $r=0.94$ for the warp), which indicates the possibility of assessing the elastic modulus of the material by speed ultrasonic waves.

As can be seen from the data presented in Figures 2-3, between the elastic modulus of the material and the speed of ultrasonic waves propagating along the layers of the material, dependences with a correlation from 0.78 to 0.95 are observed. However, the modulus of elasticity also depends on the structural characteristics of the material, such as density, porosity, or binder content. A local change in structural characteristics can lead to decreasing in the modulus of elasticity, at constant velocity of ultrasonic waves, which will lead to a larger error in assessing the modulus of elasticity. So, in [9], to assess the physical and mechanical properties of composites, it is proposed to use a multi-parameter dependence of the form:

$$
x=A \times C_{\mathrm{usw}}+B \times \frac{\mathrm{A}_{h}}{t_{h}}+C
$$

where $x$ is the required physical and mechanical characteristics; A, B, C are coefficients determined experimentally for each structure or material; $\mathrm{C}_{\mathrm{usw}}$ is the velocity of ultrasonic waves propagation along the layers of glass fabric in the direction of the preferred orientation of the filler, $A_{h}$ and $t_{h}$ are amplitude and propagation time of ultrasonic waves through thickness.

The use of the amplitude and propagation time of high-frequency $(10 \mathrm{MHz})$ ultrasonic waves as additional parameters of the model, which passed the material in the direction 
normal to the structure surface, i.e. across the layers of the filler, increases the accuracy of the relationship. This is achieved because ultrasonic waves at a frequency of $10 \mathrm{MHz}$ have a wavelength of $0.3-0.4 \mathrm{~mm}$, which increases their sensitivity to fine structural inhomogeneities.

To compare the one-parameter and multi-parameter regression dependences in the samples for bending from panel 1, we additionally measured the amplitude and propagation time of ultrasonic waves that passed the material the glass fabric layers through thickness. Based on the data obtained, one-parameter and multi-parameter dependences were constructed. Regression ratios and corrected $R^{2}$-squared values are presented in Table 2 .

Table 2. Comparison of one-parameter and multi-parameter dependencies.

\begin{tabular}{|l|l|}
\hline Regression equation & $\mathrm{R}^{2}$ adj \\
\hline$E_{\text {bend }}=0,029 \times \boldsymbol{C}_{\text {usw }}-93,44$ & 0,83 \\
\hline$E_{\text {bend }}=0,032 \times \boldsymbol{C}_{\boldsymbol{u s w}}-0,273 \times \frac{\mathrm{A}_{\boldsymbol{h}}}{\boldsymbol{t}_{\boldsymbol{h}}}-100,94$ & 0,86 \\
\hline
\end{tabular}

As can be seen from Table 2, the corrected R-square valued for the multi-parameter dependence is higher, which indicates its greater accuracy compared to the one-parameter dependence. It should be noted that in order to determine the elastic modulus by relation (5), it is necessary to carry out additional measurements of acoustic parameters through the thickness. The quality of the regression dependence can be improved by increasing the measurement accuracy, namely, by reducing the error in determining the amplitude of the ultrasonic signal caused by the instability of the acoustic contact between the surfaces of the piezoelectric transducer and the polymer composite material.

\section{Conclusion}

Significant regression dependences established on the example of fiberglass with epoxy or phenol-formaldehyde matrices reinforced with TC-8/3-K-TO glass fabric showed the possibility of evaluating the elastic modulus of polymer composite materials by the velocity of ultrasonic waves in the plane of the panels. This indicates the possibility of evaluating the elastic modulus of polymer composite materials by a non-destructive method based on the data on the velocity of ultrasonic waves propagating in the plane of the panels. This makes it possible to predict and control the operational properties of PCM structures during their manufacture and significantly reduce the likelihood of rejects. A promising direction for improving the accuracy of determining the physical and mechanical properties of polymer composite materials is the use of multi-parameter regression dependences. The use of a multiparameter dependence of the form (5) made it possible to increase the accuracy from 0.83 to 0.86. A slight increase in accuracy is associated with an error in measuring the amplitude of the ultrasonic signal caused by an unstable acoustic contact. The proposed technique for obtaining regression dependences for assessing the elastic properties of materials can be used to improve the quality of products and structural elements made of fiberglass based on various binders.

\section{References}

1. D. Ensminger and F.B. Stulen, Ultrasonics: Data, Equations, and Their Practical Uses, ISBN 978-0-8247-5830-1 (2009)

2. I.N. Ermolov, Yu. Lange, Non-Destructive Testing: Handbook: In 8 volumes, Moscow: Mechanical Engineering, (2006) (in Russian) 
3. A.I. Potapov, F.P. Pekker, Nondestructive testing of structures made of composite materials, L., "Mechanical Engineering", (1977) (in Russian)

4. D.I. Chulkov, A.V. Terekhin, M.E. Tipikin, S.I. Minin, materials of the conference "XII All-Russian conference on testing and research of properties of materials" TestMat ", Investigation of physical and mechanical properties of PCM by ultrasonic method, Moscow: VIAM State Research Center of the Russian Federation, 390 - 404, (2020) (in Russian)

5. ASTM E 1495 - 02 Standard Guide for Acousto-Ultrasonic Assessment of Composites, Laminates, and Bonded Joints

6. ISO 178:2019 Plastics - Determination of flexural properties

7. ISO 527-2:2012 Plastics - Determination of tensile properties - Part 2: Test conditions for moulding and extrusion plastics

8. S.E. Mastitsky, V. Shitikov, Statistical Analysis and Data Visualization with R, e-book, access address: http://r-analytics.blogspot.com (2014) (in Russian)

9. V.V. Murashov, Monitoring and diagnostics of multilayer structures made of polymer composite materials by acoustic methods: monograph, Moscow: Spectrum Publishing House LLC (2016) (in Russian) 